\title{
The role of figure-ground segregation in change blindness
}

\author{
ROGIER LANDMAN \\ Graduate School of Neurosciences, Amsterdam, The Netherlands \\ and The Netherlands Ophthalmic Research Institute, Amsterdam, The Netherlands \\ HENK SPEKREIJSE \\ Graduate School of Neurosciences, Amsterdam, The Netherlands \\ and \\ VICTOR A. F. LAMME \\ The Netherlands Ophthalmic Research Institute, Amsterdam, The Netherlands \\ and University of Amsterdam, Amsterdam, The Netherlands
}

\begin{abstract}
Partial report methods have shown that a large-capacity representation exists for a few hundred milliseconds after a picture has disappeared. However, change blindness studies indicate that very limited information remains available when a changed version of the image is presented subsequently. What happens to the large-capacity representation? New input after the first image may interfere, but this is likely to depend on the characteristics of the new input. In our first experiment, we show that a display containing homogeneous image elements between changing images does not render the largecapacity representation unavailable. Interference occurs when these new elements define objects. On that basis we introduce a new method to produce change blindness: The second experiment shows that change blindness can be induced by redefining figure and background, without an interval between the displays. The local features (line segments) that defined figures and background were swapped, while the contours of the figures remained where they were. Normally, changes are easily detected when there is no interval. However, our paradigm results in massive change blindness. We propose that in a change blindness experiment, there is a large-capacity representation of the original image when it is followed by a homogeneous interval display, but that change blindness occurs whenever the changed image forces resegregation of figures from the background.
\end{abstract}

Humans have a limited capacity to detect changes occurring in visual images across brief intervals (for a review on change blindness, see Rensink, 2002). Without an interval, changes are easily detectable, usually because of apparent motion effects, but an interval of $80 \mathrm{msec}$ is enough to make detection difficult (Rensink, 2002). People can monitor four to five items for change under such circumstances (Luck \& Vogel, 1997; Pashler, 1988; Rensink, 2002). This may be due to the limited capacity of working memory, which is four to five items (Luck \& Vogel, 1997).

Change blindness has been taken as evidence that our representation of the world is sparse (O'Regan \& Noë, 2001). However, partial report methods indicate that an extensive representation is available for the first few hun-

R. L. is supported by a grant from the Netherlands Organization for Scientific Research (NWO). We thank our reviewers (Greg Francis, Nelson Cowan, and Mark Becker) for their valuable comments. Correspondence should be addressed to R. Landman, Department of Visual Systems Analysis, Graduate School of Neurosciences, Amsterdam, P. O. Box 12011, 1100AA Amsterdam, The Netherlands (e-mail: rlandman@ioi.knaw.nl). dred milliseconds after an image disappears (Di Lollo \& Dixon, 1988; Loftus, Duncan, \& Gehrig, 1992; Sperling, 1960). If such a representation could be used in change detection, performance would be much better. In support of this, Becker, Pashler, and Anstis (2000) found that cuing the relevant item during the interval in a change detection task improved performance. Cuing makes it possible to transfer the relevant item from an extensive, but fleeting, sensory representation to working memory, protecting it from change blindness. A recent report has indicated that a cue remains effective at intervals of up to $1.5 \mathrm{sec}$ (Landman, Spekreijse, \& Lamme, 2003). Whereas a cue during the interval improved performance, cuing after the interval (once the changed image had appeared) was useless (Becker et al., 2000; Landman et al., 2003), suggesting that the changed image caused more interference than the interval display.

The question remains, however, why in change detection tasks an (almost) identical version of the original display, but not the interval display, interferes with memory. One causal factor may be the offset/onset of local features. According to Phillips and Singer (1974), change detection depends on the detection of a distinctive visual 
transient (a fast change in luminance or color in the retinal image) at the change location. Without an interval, the transient occurs in isolation. With the interval, local features are switched off and on, producing transients all over the screen, making the change hard to localize. A problem with this interpretation is that the interval itself produces strong transients. Yet, the interval display does not cause strong interference, since cuing is still effective during the interval (Becker et al., 2000; Landman et al., 2003).

The offset/onset of images does not only involve local features. At a higher level of description, it involves the disappearance and reappearance of objects. This could be another causal factor (O'Regan, Rensink, \& Clark, 1999). Sudden onset of objects may capture attention (Yantis \& Jonides, 1984). Moreover, simultaneously presented objects compete for processing resources (Desimone \& Duncan, 1995). Although the change event may capture attention when on its own, the signal could be swamped in the presence of concurrent attention-grabbing events (O'Regan et al., 1999).

In the present study, we investigate the role of offset/onset of local features and objects in change blindness by varying the interval display and testing the effectiveness of a cue. The first image will appear, the interval will set in, and, at one moment during the interval, a cue will appear. Next, the changed image appears. If the offset/onset of local features interferes with memory, cuing during the interval should be useless if the interval display is filled with new local features, regardless of whether these local features form objects or not. If the presence of objects is crucial, cuing should be useless only if the interval contains objects. Thus we investigate what properties an image must have in order to interfere.

\section{GENERAL METHOD}

\section{Subjects}

Seven observers ( 3 experienced, including 1 author, 4 naïve) with normal or corrected-to-normal visual acuity took part in the experiment.

\section{Materials}

The stimuli were presented on a 19-in. monitor using a PC (refresh rate, $60 \mathrm{~Hz}$ ). The observers sat at a distance of $39 \mathrm{in}$. The stimulus displays subtended $16^{\circ} \times 21^{\circ}$ of arc. Custom-written software with MGL graphics libraries displayed the stimuli on the screen and recorded the type of trial, which button was pressed, and so forth.

The stimulus displays consisted of black diagonal line segments (length, $0.5^{\circ}$ ) on a white background. The displays were filled with homogeneously oriented line segments, except at the location of figures (rectangles), where the lines had the orthogonal orientation. The rectangles (each subtending $0.9^{\circ} \times 1.8^{\circ}$ ) did not have an outline, but the texture discontinuity made them pop out from the rest of the texture, yielding the percept of figures on a background (Figure 1). Each stimulus display contained eight rectangles on an imaginary circle with a radius of $4^{\circ}$ from the center of the screen, with a random jitter for each rectangle of $0^{\circ}-1^{\circ}$ in eccentricity. Each rectangle was positioned either horizontally or vertically (randomized). Throughout the trial, there was a red fixation point (width, $0.18^{\circ}$ ) in the center of the screen.
A cue was presented to indicate which rectangle might change (the target). The cue was a yellow line, superimposed on the image currently displayed, with one end about $0.7^{\circ}$ from the fixation point and the other end about $0.7^{\circ}$ from the target.

\section{Analysis}

We used a method described by Pashler (1988) to express performance in terms of the number of items available for comparison, which we call capacity:

capacity $=\frac{(\text { hit rate } \times \text { no. figures }- \text { false alarm rate } \times \text { no. figures })}{(1-\text { false alarm rate })}$.

This formula assumes that people hold on to a particular number of items (capacity), and answer "yes" when one of them changes, whereas when there is no change among those items, they answer "no" or guess "yes" in a proportion of trials, given by the false alarm rate (Luck \& Vogel, 1997; Pashler, 1988).

In Experiment 1, we also use another version of this formula, proposed by Cowan (2001):

capacity $=($ hit rate + correct rejection rate -1$) \times$ number of items.

This formula includes the correct rejection rate, which may result in a more accurate measure, since it takes into account the possibility that subjects also use memory to determine the absence of change. As additional measures of performance, we calculated percent correct and sensitivity, $d^{\prime}$ (Green \& Swets, 1966).

Analysis of variance (ANOVA) for repeated measures was used in order to determine the existence of differences among the means. Paired $t$ tests were done to determine which means were different.

\section{EXPERIMENT 1}

\section{Method}

In Experiment 1, a change detection task was done with four types of interval display. Figure 1 shows a schematic picture of the procedure and examples of the displays. The basic procedure was as follows: A display with eight rectangles was presented for $500 \mathrm{msec}$ (Stimulus 1), then the interval display was shown for 1,600 msec, and finally, the display with the rectangles reappeared (Stimulus 2). Stimulus 1 duration was kept short to minimize shifts of attention and eye movements within the array. On $50 \%$ of the trials, one randomly chosen rectangle (target) had a different orientation in Stimulus 2 than in Stimulus 1 (horizontal/vertical). The subjects were instructed to detect whether any rectangle changed orientation across the two presentations. They were told that $50 \%$ of all trials contained a change, and one rectangle would change at a time. The subjects responded by pressing a mouse button: the right button if they thought there was a change, the left button if they thought there was no change. Stimulus 2 remained on screen until the subject responded. As the button was pressed, a high or low tone was heard, indicating a correct or incorrect response. Next, the screen was green for $1 \mathrm{sec}$ before a new trial began.

Each trial contained a cue, a yellow line flashing on the screen once, pointing at one rectangle. The meaning of the cue was explained to the subjects as follows: "If there is a change in this trial, it is the rectangle where the cue points. If that rectangle does not change, there is no change in this trial." The duration of the cue $(100 \mathrm{msec})$ was kept short to minimize interference by its own presence: Attention could be drawn to the cue itself rather than to the place at which it pointed. The rectangles that were not cued are called distractors in the text below.

Four types of interval display were used, in separate sessions. In the text below, these will be referred to as background, onedistractor, seven-distractor, or center-distractor displays (see Figure 1). The background display contained newly generated line segments, with a homogeneous orientation, orthogonal to the back- 

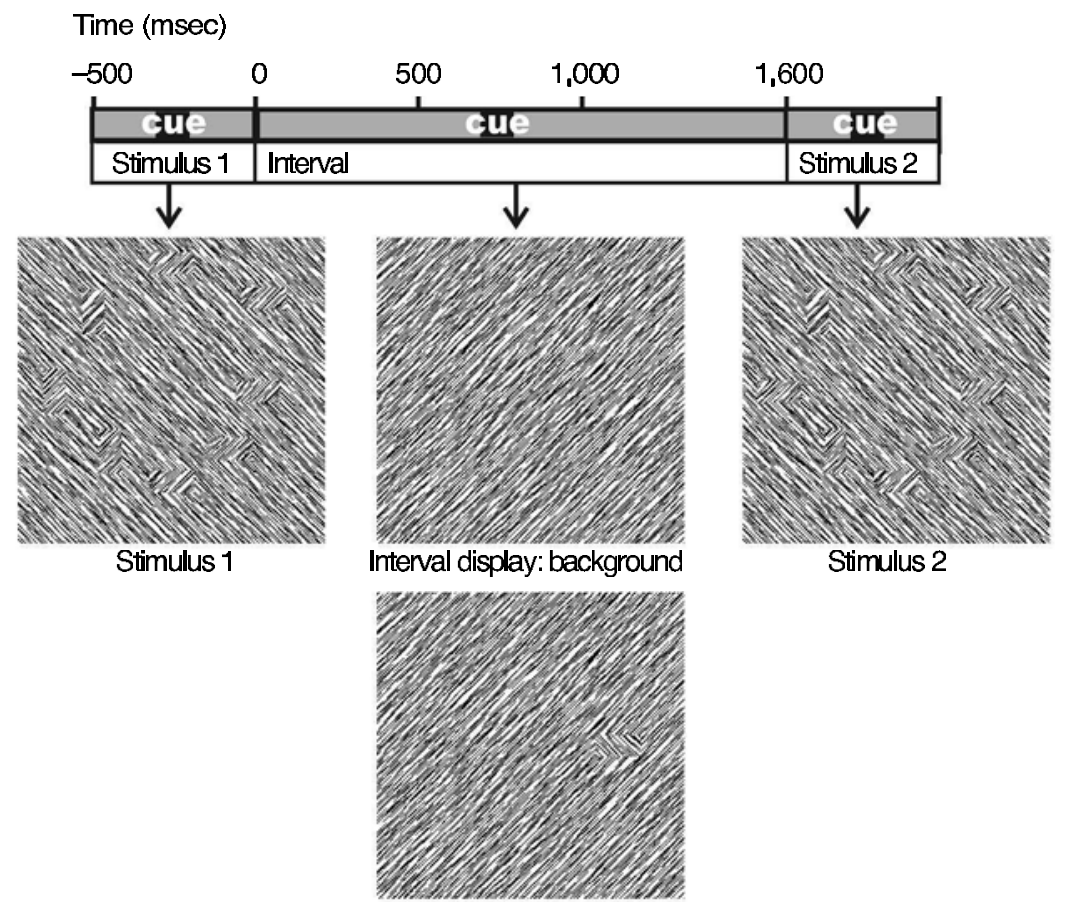

Interval display: 1 distractor

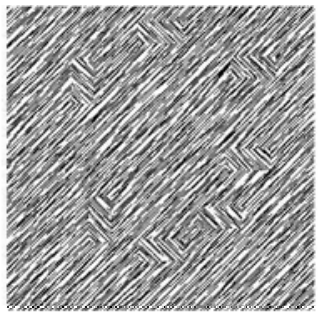

Interval display: 7 distractors

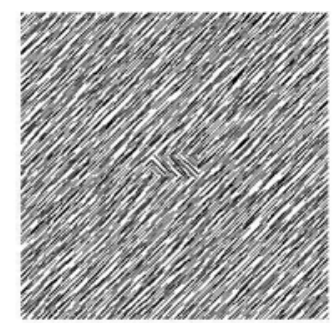

Interval display: Center distractor

Figure 1. Procedure and examples of stimulus and interval displays for the task in Experiment 1: The time bar above shows when various events start within a trial. Each trial consisted of a Stimulus 1 display, an interval display, and a Stimulus 2 display. The arrows point at examples of each. The interval display was varied, as can be seen from the examples in the figure. In the time bar, the small black bars with cue written in white indicate the possible moments at which a cue could appear. Only one cue appeared in each trial.

ground line segments in Stimulus 1. This display contained no rectangles. The one-distractor display was similar to the background display, but contained one of the distractors. This rectangle had the same orientation, size, and location as did the distractor in Stimulus 1 , except that the line segments had the orthogonal orientation. The seven-distractor display was similar to the one-distractor dis- play, but contained all rectangles except the target. Thus, the location of the target could be inferred from the locations of the seven distractors before cue appearance. The subjects were informed about this in advance. The center-distractor display was the same as the one-distractor display, except that the distractor appeared in the center of the display. 
Regardless of interval display type, the task had three conditions, based on cue onset-time, measured from Stimulus 1 offset: -300 , 600 , or $1,800 \mathrm{msec}$ (see Figure 1). The cue at $-300 \mathrm{msec}$ appeared during Stimulus 1 . The cue at $600 \mathrm{msec}$ appeared during the interval. The cue at 1,800 msec appeared $200 \mathrm{msec}$ after Stimulus 2 onset.

For each interval type, subjects ran three sessions of 48 trials, in mixed order. The percentage change/no-change trials per location and condition was 50/50. The end result contains 48 trials per cue condition. Prior to the first session, the subjects were allowed 15 practice trials.

\section{Results}

The bar charts in Figure 2 show the capacity (Pashler's formula, see Method), sensitivity $d^{\prime}$, and fraction correct, for each type of interval. These three measures show roughly the same result for the four interval types: Performance is highest with a cue before the interval, lowest with a cue after the interval, and intermediate with a cue during the interval. High performance with a cue before the interval indicates that the stimuli and cue were processed well even though they were shown for $500 \mathrm{msec}$ and $100 \mathrm{msec}$, respectively.

The difference in performance between a cue during the interval and a cue after the interval varies depending on the interval display $[F(3,18)=3.7, p=.03]$. With the background and center-distractor displays, a cue during the interval yields significantly higher capacity than a cue
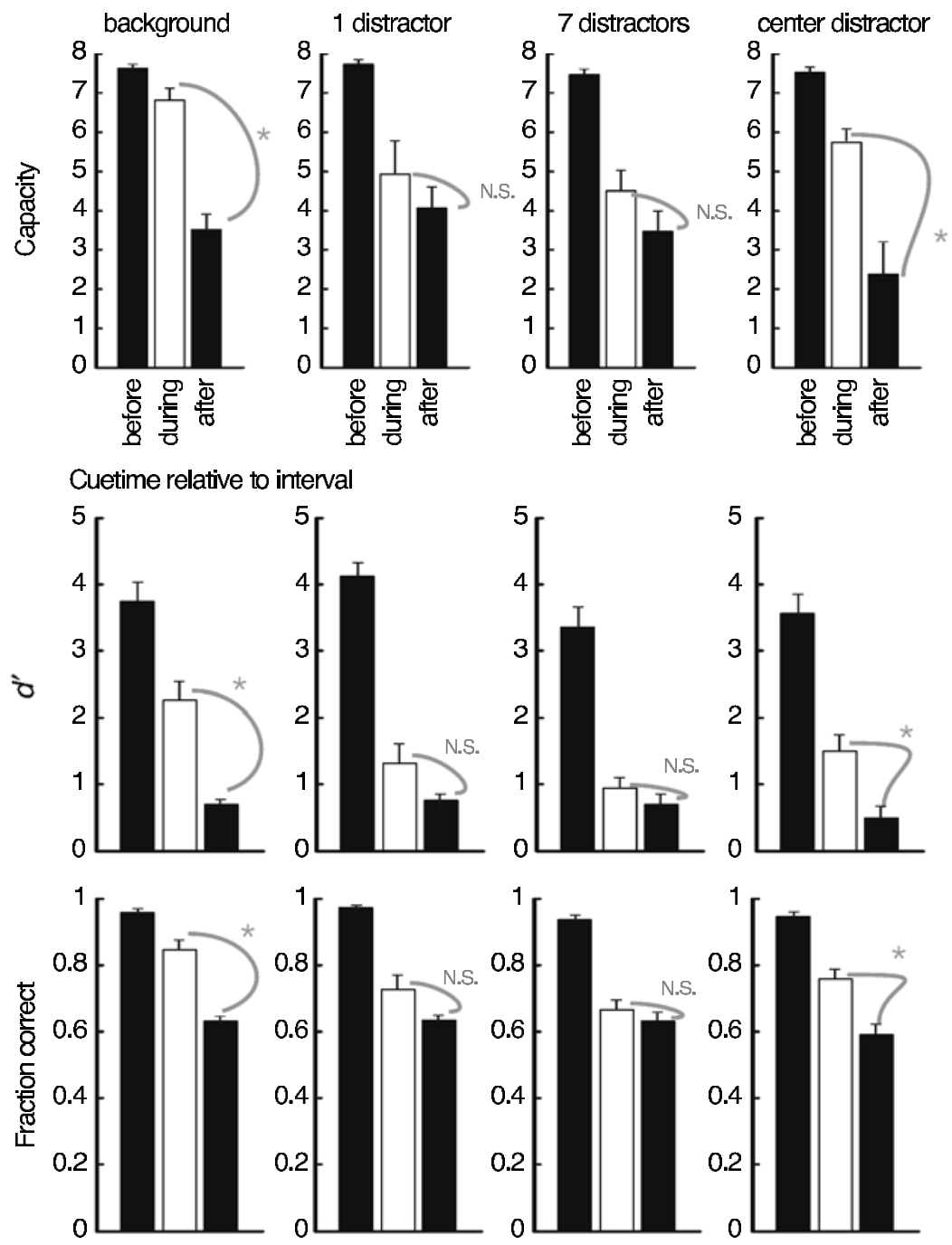

Figure 2. Results of Experiment 1: Each row of charts shows the outcome of one measure of the subjects' performance, for each type of interval display, from left to right: background, 1 distractor, 7 distractors, and center distractor. The upper row shows the outcome of the capacity measure (Pashler, 1988), the middle row shows the corresponding $d^{\prime}$ values, and the bottom row shows fraction correct. These values are on the $y$-axis. The $x$-axis in each chart indicates timing of the cue: before, during, or after the interval. Bent curves indicate the conditions that were compared in $t$ tests. Stars indicate significant differences. N.S., not significant. The sticks on top of the bars indicate the standard errors of means. 
after the interval [background, $t(6)=8.2, p=.0001$; center distractor, $t(6)=3.6, p=.01]$. With the onedistractor and seven-distractor displays, these conditions are not different $[t(6)=0.9, p=.39 ; t(6)=1.6, p=.17]$. Capacity as calculated with Cowan's formula (see Method) gave the same pattern of differences $[F(3,18)=5.49, p=$ .007 ; during vs. after background, $t(6)=6.1, p=.001$; center distractor, $t(6)=3.6, p=.01$; one distractor, $t(6)=$ $1.8, p=.12$; seven distractors, $t(6)=1.4, p=.23]$. The same pattern was obtained after excluding the subject that was author of this paper [background, $t(5)=7.9, p=.001$; center distractor, $t(5)=4.8, p=.005$; one distractor, $t(5)=0.7, p=.49$; seven distractors, $t(5)=1.5, p=.19$ ].

Our primary result, a larger capacity with a cue during than after the interval, is also observed in individual subjects. Figure 3 shows the difference in capacity between cue during and after, for each interval display, using Pashler's and Cowan's formula. Most subjects show a positive difference: larger capacity during than after the interval. According to Pashler's formula, the difference in capac- ity is almost equally large with the background and center distractor displays, yet it vanishes for the one-distractor and seven-distractor displays. A more subtle effect is observed with Cowan's formula: The largest difference is obtained with the background display, and it gradually decreases going from this display to the center distractor, one-distractor, and seven-distractor display (but note that only the first two display types give a significant difference with both formulas; see above).

We also compared differences related to interval type in the absolute sense, by comparing the white bars in Figure 2 [Pashler's formula; $F(3,18)=5.7, p=.006$ ]. Capacity during the background display is significantly higher than during the other displays [background vs. one distractor, $t(6)=2.6, p=.039$; background vs. seven distractors, $t(6)=4.7, p=.003$; background vs. center distractor, $t(6)=3.4, p=.014$ ]. This effect is as large for change trials as for no-change trials, and these conditions also differ for hit rate and correct rejections separately (all $p$ values $<.041)$. Thus, also in this analysis, the presence

Pashler:

capacity $=$ (hit rate $*$ number of figures + false alarm rate $*$ number of figures $) /(1$ - false alarm rate)
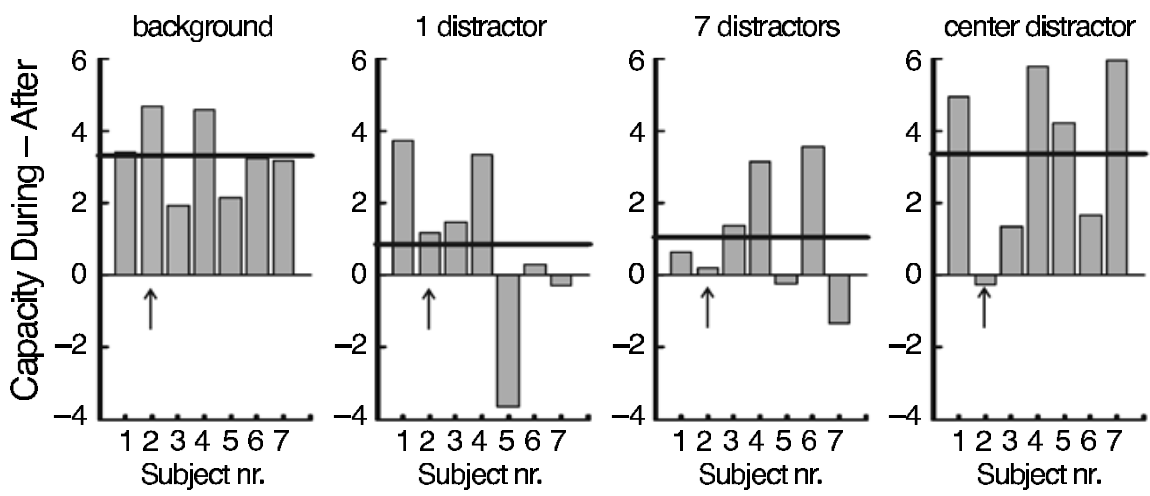

Cowan:

capacity $=($ hit rate + correct rejection -1$) *$ number of figures
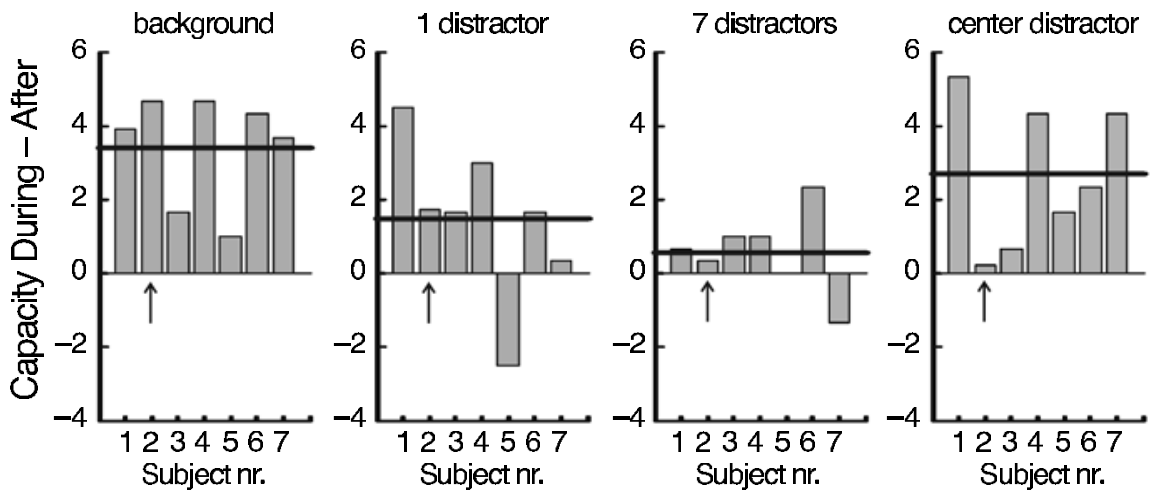

Figure 3. Difference between capacity with cue during and cue after (during minus after) for individual subjects in Experiment 1: Each bar represents a subject $(n=7)$. The upper row of charts shows the result of using Pashler's formula (Pashler, 1988); the lower row shows the result of using Cowan's formula (Cowan, 2001) (see Method). The thick horizontal lines indicate the means. The arrows indicate the author who took part as a subject. 
of objects in the interval display seems to have had a large detrimental effect on memory for the previous image. The role of object location, or number of objects, is somewhat less clearly revealed in this analysis: Of the differences between displays with distractor only, the difference between the seven-distractor and center-distractor displays is significant $[t(6)=2.9, p=.024]$.

\section{Discussion}

The results of Experiment 1 show that the effect of a cue during the interval of a change blindness task depends on the properties of the interval display. The original image, Stimulus 1, consisted of line segments defining rectangles. If the interval display contained new line segments, but no rectangles, the cue during the interval was effective, indicating the presence of a large-capacity memory representation of the image. However, when the interval consisted of not only new line segments, but also one or more rectangles, the cue during the interval was less effective. This indicates that the image appearing after the original interferes with memory more when it contains figures than when it just contains new local features.

There is some evidence that the placement of figures matters. Figures appearing at the same location as that of figures in the original image cause more interference than do figures appearing in the center. A figure in the center (center distractor) does not make a cue ineffective. This was remarkable, because according to the subjects' remarks, the center distractor was hard to ignore and it made the task more difficult. Performance with a cue during the interval was lowest with the seven-distractor display, which is also remarkable, because one could argue that this should make the task easy even without a cue: The location of the target can be inferred from the empty space among the seven distractors, and the subjects were told about this in advance. Nevertheless, the seven distractors interfered with memory.

\section{EXPERIMENT 2}

In Experiment 1, we showed that the offset/onset of local features causes more interference when these features create new rectangles at the same locations as in the original image than when these features create no rectangles. This suggests that the interference is due to the process of figure-ground segregation. What would happen if all of the rectangles remained at the same place and were just redefined? Would it also interfere, and therefore result in change blindness?

In Experiment 2, there was no interval, but we swapped the orientation of the line segments of figure and background between Stimuli 1 and 2. Thus, figures were present throughout the trial; nothing would change at the object level. Only the definition of "what makes a figure a figure" would be new. This way, the visual system is tricked into resegregating figures from background. If this resegregation does not interfere with the representation of the original image, change detection should be easy. However, if it does interfere, change detection should be difficult (i.e., the outcome of our capacity measure should indicate four items or less). That would also imply that figure-ground resegregation is a causal factor in change blindness.

\section{Method}

The general methods of this experiment were as in Experiment 1, but no interval display was used. Stimulus 1 was shown for $500 \mathrm{msec}$, then Stimulus 2 appeared. Stimulus 2 was the same as Stimulus 1, except that the orientation of the line segments (leftward slanting/rightward slanting) of figure and background were swapped, and in change trials, one of the rectangles had changed orientation (horizontal/vertical). There were four conditions, three of them based on cue onset time relative to Stimulus 1 offset: $-400,-100,200 \mathrm{msec}$, and a fourth condition with no cue. The subjects ran an equal number of trials per condition, in three sessions. The methods are illustrated in Figure 4A.

\section{Results}

It is clear from the bar chart displaying capacity that there is change blindness in this task (Figure 4B). When a cue is given in advance (first and second bar), performance is high. When the cue is given after the change (third bar), performance is significantly lower [cue $-100 \mathrm{msec}$ vs. cue 200 msec: $t(6)=5.7, p=.001$ ]. It is also lower than in the cue during condition with background interval display of Experiment $1[t(6)=5.1, p=.002)$. When no cue is given (fourth bar), performance is extremely low (capacity $\approx 1$ ).

\section{Discussion}

These results indicate that change blindness can be induced without an interval, when objects are resegregated from the background. One may argue that this is because our manipulation induced changes all over the screen. However, the background interval display in Experiment 1 included new line segments all over the screen, but the cue was still effective. What made the cue less effective in our last experiment was the fact that the new line segments redefined objects. It could also be argued that the interference occurred because people's attention followed the lines of a particular orientation and therefore shifted from targets in the first display to background in the second display. However, that implies that subjects were paying attention to the orientation of the line segments (rightward/leftward slanting), rather than the orientations of the rectangles (horizontal/vertical), when only the latter were relevant in this task. Therefore, unless the line segments captured attention, which we think is unlikely, this explanation cannot account for our results.

After the change, a cue still produces an advantage over getting no cue at all, possibly because the cue restricts comparison to a single item, whereas without a cue, the entire array needs to be compared. However, cue after is lower than cue before, and also lower than cue during compared with performance with the background display in Experiment 1, indicating that our hypothesis is correct: Resegregation of the figures causes significant interference. 

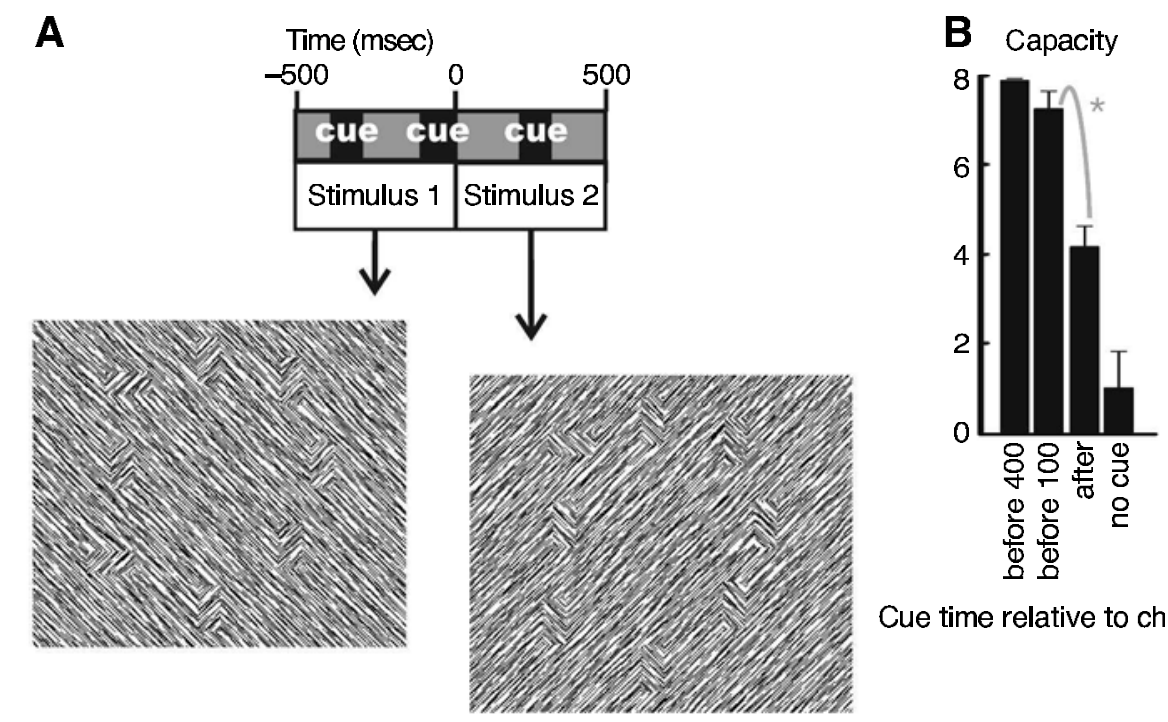

Cue time relative to change

\begin{abstract}
Figure 4. Procedure, stimuli, and results from Experiment 2: (A) Procedure and examples of stimulus displays. The time bar shows when various events started within a trial. Each trial consisted of a Stimulus 1 display and a Stimulus 2 display. The arrows point at examples of each. The small black bars with cue written in white indicate the possible moments at which a cue could appear. One cue appeared in each trial, and sometimes no cue appeared. (B) Results. The chart shows capacity (Pashler, 1988) on the $y$-axis. On the $x$-axis are the conditions in the task, based on whether, and when, the cue appeared: $400 \mathrm{msec}$ before the change, $100 \mathrm{msec}$ before the change, after the change, or not at all (the no-cue condition). Bent curves indicate the conditions that were compared in a $t$ test. The star indicates significant difference. The sticks on top of the bars indicate the standard error of mean.
\end{abstract}

\section{GENERAL DISCUSSION}

We asked people to do a change detection task and introduced various visual stimuli in the interval between the original and the changed stimuli. A cue was given, indicating which item might change. When the interval contained new local features all across the display but no objects, a cue during the interval improved change detection, indicating the presence of a large-capacity representation during the interval. When the interval display contained one distractor, or seven distractors, there was no cue advantage, indicating that much of the representation is lost when figures are newly segregated from the background. The role of figure-ground segregation was confirmed by showing that change blindness occurs when figures are redefined by new local features.

Our results confirm the findings of Becker et al. (2000) in showing that a cue during the interval enables subjects to transfer the relevant item from a large, but fragile, store to working memory and complete the task successfully. The effectiveness of a cue during the interval is likely to be due to the presence of a large-capacity, but fragile, memory trace. Upon arrival of the cue, the cued item is transferred to a more durable store (Sperling, 1960). The large-capacity representation of the original image may be sensory memory. However, sensory memory lasts no longer than a quarter of a second (Di Lollo \& Dixon, 1988; Loftus et al., 1992), whereas in the present study, the cue was advantageous at $600 \mathrm{msec}$, and in a previous study, even at $1.5 \mathrm{sec}$ after stimulus offset (Landman et al., 2003). The memory that was used to take advantage of the cue in our experiments may be of a different kind. It has been proposed that there are two types of sensory memory (Cowan, 1995): a brief, literal afterimage, and a longer sensory memory that involves more deeply processed stimuli and lasts $2-4 \mathrm{sec}$. Our results are compatible with the second type. However, the present experiments were not specifically designed to establish whether the memory trace has "sensory" properties. It could be argued that this is just working memory, although that would mean that working memory contains more than the usual four items (Cowan, 2001), at least during the first second after stimulus offset. It would also mean that during this period, working memory is very susceptible to interference.

It has been argued that change blindness indicates the absence of a prechange representation of the relevant item (O'Regan \& Noë, 2001). However, our results show that there is a large-capacity representation after the prechange image has disappeared. This representation can be interfered with, but the interference is not produced by just any new stimulus. A display with homogeneously oriented new line segments, creating visual transients all over the screen, leaves the representation intact. The presence of objects appears to be more important. When figures are resegregated from the background at the same location as that of those in the original image, the original representation is impaired. In 
"gap-contingent paradigms" (Rensink, 2002), a homogeneous display is usually shown during a temporal gap between the original and the altered stimulus, and the onset of the changed image forces resegregation of figures from ground. We hypothesize that resegregation is a causal factor in change blindness, at least in gapcontingent paradigms. Additional factors may be involved in other conditions, as when the change occurs gradually over the course of several seconds (Simons, Franconeri, \& Reimer, 2000). Finally, the present results leave open the possibility that concurrent attentiongrabbing events make a change hard to isolate (O'Regan et al., 1999), but suggest that visual transients per se are not sufficient. To further elucidate the relative involvement of transients, figure-ground segregation, and attention in change blindness, more research is required.

\section{REFERENCES}

Becker, M. W., Pashler, H., \& Anstis, S. M. (2000). The role of iconic memory in change detection tasks. Perception, 29, 273-286.

CowAn, N. (1995). Attention and memory: An integrated framework. New York: Oxford University Press.

Cowan, N. (2001). The magical number 4 in short-term memory: A reconsideration of mental storage capacity. Behavioral \& Brain Sciences, 24, 87-114.

Desimone, R., \& Duncan, J. (1995). Neural mechanisms of selective visual attention. Annual Review of Neuroscience, 18, 193-222.

Di Lollo, V., \& DiXon, P. (1988). Two forms of persistence in visual information processing. Journal of Experimental Psychology: Human Perception \& Performance, 14, 671-681.
Green, D. M., \& Swets, J. A. (1966). Signal detection theory and psychophysics. New York: Wiley.

Landman, R., SPekreiJse, H., \& LAmme, V. A. F. (2003). Large capacity storage of integrated objects before change blindness. Vision Research, 43, 149-164.

Loftus, G. R., DunCAn, J., \& Gehrig, P. (1992). On the time course of perceptual information that results from a brief visual presentation. Journal of Experimental Psychology: Human Perception \& Performance, 18, 530-549.

LUCK, S. J., \& VOGEL, E. K. (1997). The capacity of visual working memory for features and conjunctions. Nature, 390, 279-281.

O'REGAN, J. K., \& NOË, A. (2001). A sensorimotor account of vision and visual consciousness. Behavioral \& Brain Sciences, 24, 939-973.

O'Regan, J. K., RensinK, R. A., \& ClaRK, J. J. (1999). Change-blindness as a result of "mudsplashes." Nature, 398, 34.

PAShler, H. (1988). Familiarity and visual change detection. Perception \& Psychophysics, 44, 369-378.

Phillips, W. A., \& Singer, W. (1974). Function and interaction of on and off transients in vision: I. Psychophysics. Experimental Brain Research, 19, 493-506.

Rensink, R. A. (2002). Change detection. Annual Review of Psychology, 53, 245-277.

Simons, D. J., Franconeri, S. L., \& REIMER, R. L. (2000). Change blindness in the absence of visual disruption. Perception, 29, 1143-1154.

SPERLING, G. (1960). The information available in brief visual presentations. Psychological Monographs, 74 (11, Whole No. 498), 1-29.

YANTIS, S., \& JONIDES J. (1984). Abrupt visual onsets and selective attention: Evidence from visual search. Journal of Experimental Psychology: Human Perception \& Performance, 10, 601-621.

(Manuscript received August 14, 2002; revision accepted for publication April 7, 2003.) 